\title{
28 Research Square \\ Sterile water; a novel and promising human experimental muscle pain model
}

Sofia Louca Jounger ( $\square$ sofia.louca@ki.se )

Karolinska Institutet https://orcid.org/0000-0001-8391-5991

Johanna Svedenlöf

Karolinska Institutet

Reija Elenius

Karolinska Institutet

Christoffer Källkrans

Karolinska Institutet

Emil Scheid

Karolinska Institutet

Malin Ernberg

Karolinska Institutet

Nikolaos Christidis

Karolinska Institutet

Research article

Keywords: Sterile water, Experimental pain model, Hypertonic saline, Pain, Masseter muscle

Posted Date: May 8th, 2020

DOI: https://doi.org/10.21203/rs.3.rs-26494/v1

License: (c) (1) This work is licensed under a Creative Commons Attribution 4.0 International License.

Read Full License 


\section{Abstract}

Background Intramuscular injection of hypertonic saline evokes pain with similar characteristics as clinical myalgia and is thus, considered a valid human experimental model. The aim of this study was to investigate if intramuscular injection of sterile water can be used as a human experimental pain model by comparing it with hypertonic and isotonic saline, and to analyze if the effects differ between men and women. Methods This randomized double blind and placebo-controlled study included 15 healthy women and 15 healthy age-matched men (mean (SD) age of 23.6 (2.4) years). The study comprised of three separate sessions, with at least one week of wash out between each session. Sterile water (i.e. the testsubstance), hypertonic saline (active control), and isotonic saline (passive control) were injected intramuscularly into one of the masseter muscles in a randomized order. Pain intensity (VAS) was assessed every 15 th $\mathrm{s}$ after the injection and pain duration (s) as well as pain drawing area (au) were recorded. Pressure pain thresholds $(\mathrm{kPa})$ were assessed every $5 \mathrm{~min}$ after injection during $30 \mathrm{~min}$. Results All substances evoked pain $(P<0001)$, but sterile water and hypertonic saline induced pain with higher intensity $(P<0.001)$, longer duration $(P<0.001)$, and larger pain drawing area $(P<0.001)$ than isotonic saline. The pain intensity was higher for hypertonic saline than sterile water $45-165 \mathrm{~s}$ after injection $(\mathrm{P}<$ 0.015), but there were no significant differences between them regarding maximum pain intensity, pain duration or pain drawing area. There was no significant difference in PPT values with time or between substances. The pain duration was longer in the men compared to the women for all substances $(\mathrm{P}<$ 0.006), while the pain drawing area was larger in women after injection of hypertonic saline $(P<0.003)$, but not after injection of the other substances. No other sex differences were found. Conclusion Injection of sterile water had similar effects as hypertonic saline on pain variables, but no effect on PPT. Hence, the model mimics clinical myalgia and may offer a novel and simpler alternative to hypertonic saline injections.

\section{Background}

Painful temporomandibular disorder (TMD) is the second most common musculoskeletal disorder after chronic back pain $(1,2)$, affecting up to $5-12 \%$ of the adult population and twice as many women as men $(3,4)$. TMD includes conditions such as chronic muscular and joint pain, impaired jaw function, as well as articulating sounds from the temporomandibular joint. TMD myalgia is often described as a dull, pressing muscle pain of moderate intensity, which can be intensified to a more intense and sharp pain when provoked (5). Studies have shown that chronic pain of moderate to severe intensity requires specific care and treatment $(1,6)$. Further, patients with TMD myalgia often report pain upon chewing, mouth opening difficulties as well as headache, but also suffer from depression and anxiety (7-9). Taken together, TMD myalgia affects the quality of life negatively (1).

To investigate pathophysiological aspects and therapeutic approaches of painful TMDs in a standardized situation to minimize confounding factors, one have to mimic the clinical setting using experimental pain models $(10,11)$. In other words, the experimental pain model should: a) evoke pain in a controlled situation; b) be fully reversible; $c$ ) be homogenous across participants; $d$ ) short lasting (but 
long enough to evaluate specific pathophysiological aspects and therapeutic approaches as well as facilitating assessment of various pain effects on the sensory-motor system) $(10,12)$.

In experimental pain models nociceptors in the structure that will be investigated, such as muscles and joint, are activated (13). After the stimulus is administered pain pathways and pain characteristics can be assessed, but also therapeutic agents as well as the effect pain has on function can be studied $(14,15)$. There are several types of stimuli used and they can be divided into two groups; endogenous and exogenous. The endogenous pain models use a natural stimulus such as ischemia or exercise to induce muscle pain (15). Exogenous pain models, on the other hand, use external stimuli such as thermal, mechanical and chemical. Injections with glutamate, hypertonic saline, acidic saline, serotonin, bradykinin, and nerve growth factor (NGF) have been used to chemically induce muscle pain and/or allodynia/hyperalgesia (14-18). However, all these models have limitations. Hence, none of them can fully mimic the actual pain experience of chronic myalgia. For example, hypertonic saline induces shortlasting (minutes) sharp and deep pain that resembles clinical myalgia, but with a higher intensity $(11,15$, 19) and no allodynia/hyperalgesia. So, finding an exogenous experimental pain model that better mimics the characteristics of clinical myalgia could broaden the knowledge of the pathophysiological aspects as well as increase the trustworthiness of the findings when investigating new therapeutic approaches.

Cutaneous injection of sterile water into the lower back region is commonly used during labor with the purpose to direct pain from internal organs to the skin (20-22). The cutaneous injection evokes a sharp pain sensation which in turn is believed to activate the conditioned pain modulation (CPM) pathways to inhibit organ pain during labor (23). Due to its hypotonic sodium content, water generates an osmotic gradient which causes intense pain in biological tissues $(24,25)$. When compared to other chemical substances as well as isotonic saline injected intramuscularly, studies in rabbits and rodents have shown that sterile water causes less muscle necrosis and hemorrhage $(22,26)$. Therefore, sterile water injection into muscles could perhaps offer an alternative to other human experimental pain models, but to the best of our knowledge, this has not been investigated. In contrast to other substances it is cheap and does not require production on demand by a pharmacy (glutamate, acidic saline) or dilution before injection (hypertonic saline).

Therefore, the aim of this study was to test the hypothesis that intramuscular injection of (hypotonic) sterile water can be used as a human experimental pain model for clinical masseter myalgia by comparing it with hypertonic and isotonic saline. A second aim was to analyze if the effects differ between men and women.

\section{Materials And Methods}

The study followed the present guidelines according to the Declaration of Helsinki and was approved by the Regional Ethical Review Board in Stockholm, Sweden (2019/3:1). The experiments were conducted at the clinical research lab of the Department of Dental Medicine, Karolinska Institutet, Huddinge, Sweden 
between February 2019 to May 2019. Verbal and written information of the study was provided to all participants and their written consent was obtained before the start of study.

\section{Participants}

The study comprised of fifteen healthy women with a mean (SD) age of 23.5 (1.9) years and 15 healthy age-matched men with a mean (SD) age of 23.7 (2.9) years. The volunteers were recruited by flyers placed at different floors at the Department of Dental Medicine, Karolinska Institutet, as well as Södertörn University, both in Huddinge, Sweden.

The inclusion criteria were; 1) age between 18 to 40 years; and 2) good general health. Participants were excluded if they had; a) any current pain from the orofacial region; b) a pain diagnosis of TMD according to the Diagnostic Criteria for TMD (DC/TMD)(3); c) any type of headache; d) diagnosed systemic muscular and/or joint diseases, such as fibromyalgia or rheumatoid arthritis; e) whiplash-associated disorders; f) neuropathic pain or neurological disorders; g) severe psychiatric conditions (diagnosed by a physician); h) pregnancy or lactation; i) use of any kind of medication except for contraceptives 48 hours preceding the study day; and $\mathbf{j}$ ) any previous negative reactions to either of the substances to be injected.

\section{Study protocol}

The study protocol had a randomized, double blind and placebo-controlled design, where each participant was his/her own control. The study comprised of three separate sessions, with at least one week of wash out between each session (Fig. 1). The sessions, in which sterile water (test-substance), hypertonic saline (active control), and isotonic saline (passive control) were injected into the masseter muscle were in a random order. To randomize the order of injections a randomization list was generated using a webbased randomization tool (www.randomization.com, seed 8010) by one of the researchers not participating in data collection (NCh).

The participants were seated in the same conventional dental chair, in a quiet environment, during all three sessions. The participants first completed a questionnaire containing screening instruments for stress (Perceived Stress Scale; PSS-10), depression (Patient Health Questionnaire; PHQ-9), pain catastrophizing (Pain Catastrophizing Scale-13; PCS-13), anxiety (Generalized Anxiety Disorder Scale-7; GAD-7), and nonspecific physical symptom (Patient Health Questionnaire; PHQ-15), all included in the DC/TMD Axis II protocol (3). The women were asked about at which day of the menstrual cycle she was, counted from the first day of the last menses. The menstrual cycle was divided into five phases: menstrual (days 1-5), follicular (days 6-11), pre-ovulatory (days 12-16), luteal (days 17-23), and premenstrual (days 24-28) (27). A clinical examination according to the DC/TMD Axis I protocol followed for trial suitability and to ensure that the participants did not meet any of the exclusion criteria. Baseline recordings of pain characteristics and pressure pain threshold (PPT) were conducted. After the baseline recordings injection of one of the substances was done, where after pain assessments and recordings of PPT were repeated.

\section{Injections}


The substances used were: 1) sterile water (0 mOsm/l; B. Braun Melsungen AG, Melsungen, Germany) (28); 2) hypertonic saline $(\mathrm{NaCl} 58.5 \mathrm{mg} / \mathrm{mL})$ that was prepared from a concentrated solution of sodium chloride ( $\mathrm{NaCl} 234 \mathrm{mg} / \mathrm{mL}, 2002 \mathrm{mOsm} / \mathrm{l}$; B. Braun Melsungen, Germany) by mixing it in a 1:3 proportion with sterile water (B. Braun Melsungen AG, Melsungen, Germany) shortly before injection; and 3 ) isotonic saline ( $\mathrm{NaCl} 9 \mathrm{mg} / \mathrm{mL}, 308 \mathrm{mOsm} / \mathrm{l} ; \mathrm{B}$. Braun Melsungen AG, Melsungen, Germany). Preparation and blinding of injection substances was made by one of the researchers (JS, ES, NCh) not participating in data collection. All liquids were clear, colorless, and odorless. The syringes therefore appeared identical making it impossible for the researchers collecting and registering the data (RE, CK), and for the participants to differentiate one substance from another.

The most prominent point of the right masseter muscle was used for injections (in the midline, approximately $2 \mathrm{~cm}$ superior to the mandibular border). This point was determined by manual palpation during contraction of the muscle. The skin over the injection site was cleaned with an injection swab (B. Braun, Isopropyl Alcohol 70\%) and left to dry for at least 30 seconds before injection. A needle with a diameter of $0.4 \mathrm{~mm}$ and a length of $19 \mathrm{~mm}$ was used. The needle was inserted into the relaxed muscle to a depth of approximately $15 \mathrm{~mm}$ and $0.2 \mathrm{~mL}$ of each substance was injected during $10 \mathrm{sec}$.

\section{Assessment of pain characteristics}

A 0-100 mm visual analog scale (VAS) was used for assessment of pain intensity. The scale was drawn as lines on sheets pf paper that the participant had in front of him/her. The end points were marked with "no pain" on the left side and "worst pain experienced" on the right side (29). Pain intensity was assessed directly after injection (time 0 ) and then every 15 th second until pain had subsided or a maximum of 5 min. If pain persisted 5 min after injection the participant was instructed to inform the experimenter when it had subsided, and this time-point was recorded. From these data the maximal pain intensity (peak pain intensity), pain duration (s), and the area under the curve (VAS auc), i.e. the cumulated pain intensities multiplied by the pain duration (expressed in arbitrary units, au) were extracted and used in the analysis.

Pain drawings were used to assess the distribution (spread) of pain caused by the injection, i.e. the pain area. Five min after injection or when pain had subsided, the participants were asked to encircle the maximal pain distribution on one extra-oral lateral view of the head and on one lateral view of the head highlightening the jaws and teeth (both on the side of injection). All pain drawings were scanned separately using the printer Ricoh MP C6004ex with a resolution of $300 \mathrm{dpi}$, and the pain area was calculated (au) using an area calculation function in a photo editing program (Adobe Photoshop CC2019, Adobe Systems Incorporated, USA).

\section{Assessment of pressure pain threshold}

PPT was recorded $(\mathrm{kPa})$ using an electronic pressure algometer (Somedic Sales AB, Hörby, Sweden) with a $1 \mathrm{~cm}^{2}$ probe tip covered with a $1 \mathrm{~mm}$ thick rubber disc. The pressure algometer was placed perpendicular to the skin overlaying the masseter muscle at the most prominent point of the masseter muscle. The tip of the right index finger was used as reference point. The rate of increase of pressure was 
pre-set to $50 \mathrm{kPa} / \mathrm{s}$. When the participants perceived that the sensation of pressure turned into pain, they pressed a signal button to stop the increase of pressure. The participants were instructed beforehand and to ensure correct procedure, the PPT was first practiced the recording on the dorsal side on the right thumb. The pressure algometer was balanced after each measurement. The PPT was assessed at baseline, immediately after injection, and then at 5, 10, 15, 20, 25 as well as 30 min after injection. Baseline PPT was calculated as the mean value of three assessments, while the PPT at the other time points was calculated as the mean value of two assessments (to reduce the risk of temporal summation).

\section{Data analysis and statistics}

The SigmaPlot for Windows version 14.0 software (Systat Software Inc., San Jose, CA, USA) was used for data analysis. The Shapiro-Wilk's test was used to test the normality of the data. Parametric statistical methods were used for normally distributed data, while non-parametric statistics were used for data that were not normally distributed. Mean and standard deviation (SD) were used for descriptive statistics for normally distributed data, while median and interquartile range (IQR) were used for descriptive statistics of non-normally distributed data.

Data regarding pain intensity and PPT were normally distributed. Therefore, two-way RM ANOVA (2-way RM ANOVA) with substance as the independent factor and time as the repeated factor was used to analyze differences over time. When the RM ANOVA indicated a significant time difference, the Tukey test for multiple comparison versus a control group (baseline) was used as a post-hoc test, and to test differences between substances and interactions at the different time points. The PPT values were normalized to baseline, i.e. the relative changes (\%) were used in the statistical analyses.

Data regarding peak pain intensity, pain duration, VAS auc, and pain area from pain drawings were not normally distributed. An attempt to log transformation did not change this so these data were analyzed using non-parametric methods. The Friedman repeated measures (RM) analysis of variance (ANOVA) on ranks was used to test for differences between substances. When a significant difference was indicated, the Dunn's method for multiple comparisons versus a control group (isotonic saline) was used as a posthoc test. Sex differences for these variables were analyzed with Mann-Whitney U-test.

The significance level was set to $P<0.05$ for all tests.

\section{Results}

All 30 participants completed all three sessions, thus there were no dropouts. None of the participants reported any adverse event for any of the included test substances. Further, there were no significant sex differences in any of the background variables or psychosocial scores according to the Axis II questionnaire (Table 1). All participating women were in different phases of their menstrual cycle independently of injected substance.

\section{Pain characteristics}




\section{Pain intensity}

The 2-way RM ANOVA showed a time effect ( $\mathrm{df}=21 ; \mathrm{F}=84.053 ; P<0.001)$, a difference between substances $(\mathrm{df}=2 ; \mathrm{F}=69.226 ; P<0.001)$, and an interaction between time and substance $(\mathrm{df}=42 ; \mathrm{F}=$ 38.481; $P<0.001)$. The post-hoc test showed that sterile water evoked pain that was higher than at baseline at all time-points after injection, i.e. 0-300 s $(P<0.001$, Tukey test), while hypertonic saline evoked pain of higher intensity compared to baseline $0-285 \mathrm{~s}$ after injection ( $P=0.022$, Tukey test). Isotonic saline, on the other hand, only evoked pain of higher intensity than baseline during the first $15 \mathrm{~s}$ after injection ( $P=0.024$, Tukey test). Finally, when the substances were compared, hypertonic saline induced pain with a higher intensity than sterile water 0 and 30-165 s $(P<0.015$, Tukey test) and isotonic saline $0-285 \mathrm{~s}$ after injection $(P<0.036$, Tukey test). Sterile water induced pain with a significantly higher intensity than isotonic saline 0-300 s after injection ( $P<0.001$, Tukey test), all shown in Fig. 2.

\section{Peak pain intensity, pain duration, and AUC}

The Friedman RM ANOVA showed that peak pain intensity differed significantly between substances $(P<$ 0.001). The post-hoc test showed that it was higher after injection of both sterile water and hypertonic saline when compared to isotonic saline ( $P<0.001$, Dunn's method). However, there was no difference in peak pain intensity between sterile water and hypertonic saline.

The Friedman RM ANOVA showed that the pain duration differed significantly between substances $(P<$ $0.001)$. The post hoc test showed that it was longer after injection of sterile water $(P<0.001$; Dunn's method) and hypertonic saline ( $P<0.001$; Dunn's method) when compared to isotonic saline. Although sterile water induced pain with a duration that was twice as long $(220 \%)$ than hypertonic saline, this difference was not significant ( $P=0.082$; Dunn's method), as shown in Table 2.

The VAS auc followed the same pattern as the evoked changes in peak pain intensity and pain duration. The Friedman RM ANOVA showed that it differed significantly between substances $(P<0.001)$. VAS auc was significantly larger after injection of sterile water $(P<0.001$; Dunn's method) and hypertonic saline ( $P$ $<0.001$; Dunn's method) when compared isotonic saline. However, there was no significant difference between sterile water and hypertonic saline ( $P=1.000$; Dunn's method), shown in Table 2.

\section{Pain area (spread)}

The Friedman RM ANOVA showed that the induced pain area after injections differed between substances $(P<0.001)$. The pain area was larger after injection of sterile water when compared to isotonic saline ( $P<0.001$; Dunn's method). Also, hypertonic saline induced a pain area that was larger than isotonic saline $(P<0.001$; Dunn's method), but there was no significant difference between sterile water and hypertonic saline, as shown in Table 2.

\section{Pressure pain threshold}

None of the substances affected the PPT. The 2-way RM ANOVA showed no significant time effect ( $\mathrm{df}=6$; $\mathrm{F}=0.525 ; P=0.789)$, no difference between substances ( $\mathrm{df}=2 ; \mathrm{F}=0.0849 ; P=0.919)$, and no interaction 
between time and substances ( $\mathrm{df}=12 ; \mathrm{F}=1.520 ; P=0.115)$, as shown in Fig. 3a. Also, there were no significant changes in the PPT over the reference point, as shown in Fig. $3 \mathrm{~b}$.

Since the men and women displayed a somewhat different profile in PPT changes after injections, data were also analyzed for each sex separately. In the women, the 2-way RM ANOVA showed a significant time effect $(\mathrm{df}=6 ; \mathrm{F}=3.607 ; P=0.003)$, but no differences between substances $(\mathrm{df}=2 ; \mathrm{F}=0.0816 ; P=$ $0.922)$ or any interaction between time and substances ( $\mathrm{df}=12 ; \mathrm{F}=1.771 ; P=0.057)$. The post-hoc test revealed that PPT was significantly lower $5 \mathrm{~min}$ after injection of hypertonic saline compared to baseline ( $P=0.003$, Tukey test $)$. In the men, there were no significant time effect ( $d f=6 ; \mathrm{F}=0.804 ; \mathrm{p}=0.569)$, difference between substances ( $\mathrm{df}=2 ; \mathrm{F}=0.144 ; P=0.866)$, or interaction between time and substances (df $=12 ; \mathrm{F}=0.441 ; P=0.944)$ after injections, all shown in Fig. 3.

\section{Sex differences}

There were no sex differences in peak pain intensity for sterile water, hypertonic saline or isotonic saline. Women displayed a significantly shorter pain duration than men after injection of all substances. Women further reported a significantly larger pain area than men after injection of hypertonic saline $(P=0.003)$ but not after sterile water or isotonic saline. There were no differences in VAS auc between sexes for any of the substances (Table 2).

\section{Discussion}

To our knowledge, this study is the first in which intramuscular injections of sterile water were investigated scientifically in humans. The main findings were that injections of sterile water into the masseter muscle induced similar pain intensity (peak pain intensity), pain duration, VAS auc, and pain area as hypertonic saline i.e. significantly higher pain intensity, longer duration and larger pain spread compared to isotonic saline. These findings suggest that sterile water can be used as an alternative acute experimental pain model to hypertonic saline. However, in similarity to hypertonic saline, sterile water did not affect the PPT, why it does not fully mimic clinical masseter myalgia.

Intramuscular injections of sterile water caused a short-lasting pain of a moderate intensity. This is in line with other commonly used experimental pain models, such as hypertonic saline or glutamate injections into the masseter muscle that cause pain less than 10 min with an average peak-pain intensity around NRS 5-6 $(10,16,30)$. However, sterile water had a somewhat different pain profile than hypertonic saline. Peak pain intensity was reached directly after injection and then the pain immediately started to decline but with a slower rate than hypertonic saline. Hypertonic saline, on the other hand, reached its peak pain intensity after about one min after injection. The pain then stayed on a higher level than for sterile water for approximately $1.5 \mathrm{~min}$. There were both similarities and differences between sterile water and hypertonic saline in pain characteristics. For example, both sterile water and hypertonic saline evoked pain of significantly higher intensity and pain area when compared to isotonic saline. Regarding hypertonic saline, this is in accordance with previous studies $(11,31,32)$. On the other hand, sterile water induced pain with a $120 \%$ longer duration than hypertonic saline (NS), which is an advantage over 
hypertonic saline. Another advantage is that sterile water is bought ready-made in sterile plastic ampules, while hypertonic saline needs to be diluted to the correct concentration in the clinic/laboratory. This possesses a risk for varying concentrations and the sterility of the solution.

In contrast to the effect on pain variables, our findings showed no significant changes in PPT values after injections of any substance. Also regarding PPT after hypertonic saline injection, previous studies show contradictory results. A few studies report reduced muscle PPT, i.e. mechanical sensitization $(11,33)$, while other studies report no mechanical sensitization $(10,18,34-37)$. These findings indicate that experimentally induced muscle pain with hypertonic saline do not necessarily lead to muscle sensitization. Based on the findings from this single study it seems that sterile water shows the same lack of mechanical sensitization. This is a disadvantage for an experimental model for clinical myalgia. Nevertheless, these results together show that sterile water may be used as an alternative experimental pain model to hypertonic saline (10).

The pain mechanism for hypertonic saline is not clear but is theorized that pain is caused by a shift in sodium concentrations leading to depolarization of excitable membranes, which in turn cause free muscle afferents to be activated by a higher osmolality $(19,38)$. In a previous study, sodium concentrations were recorded to be $40.5 \%$ higher after injections of hypertonic saline (39). Hypertonic saline has an osmolarity of $2002 \mathrm{mOsm} / \mathrm{I}$ which is higher than that of plasma (308 mOsm/l) (28). According to other studies, solutions with different osmolarity than blood irritate biological tissues and thus, may cause pain $(24,25)$. Similar, a shift in the osmolarity may explain the pain evoked by sterile water. However, sterile water is a hypotonic solution with an osmolarity lower than plasma (28). When a hypotonic solution is injected in biological tissues pain will occur due to a lower osmolarity as well as a lower osmotic pressure (40). After injection of a hypotonic saline solution, water from the extracellular fluid flows into the cell causing it to swell. This influx of water is due to a change in ion concentrations as well as a change in osmotic pressure (41). To restore the osmotic gradient balance, sodium and potassium channels are opened and an outflow of sodium and potassium will occur. The ions will then affect the muscle afferents to induce pain. When the osmotic imbalance is restored the cell will return to its normal size (42).

There were some sex differences regarding pain characteristics with a longer pain duration in men for all substances and larger pain area in women after experimentally induced pain by hypertonic saline.

However, there were no differences between sexes in peak pain intensity or VAS auc. Earlier studies report inconsistent results regarding sex differences in pain characteristic after algesic injections. For example, in one study women had a longer pain duration and larger pain area than men after experimentally induced muscle pain with acidic saline (17). Yet other studies report no differences in pain duration (11, $31)$, but larger pain area in women after hypertonic saline injection $(17,31,43)$. Furthermore, although results from this study report no differences in peak pain intensity, other experimental studies show that women rate pain higher than men after glutamate-induced pain in the masseter muscle $(16,44)$. One possible explanation could be the differences in the particular group of individuals included in this study i.e. the group was somewhat younger compared to previous studies $(11,17,31)$, hence with less or no 
previous pain experiences in life. Since most experimental studies also include quite few participants sample selection probably could explain the somewhat different results across studies.

PPT did not differ significantly between sexes after injections, although PPT decreased by $20 \% 5$ minutes after injection of sterile water in women. Hence, the results are in concordance with a few studies that reported no sex difference in PPT after hypertonic saline injection $(11,34)$, and glutamate injection (16, 44). However, the PPT values were generally higher in men, which supports results from some previous studies of sex differences in masseter PPT $(45,46)$. Taken together, our results regarding sex differences support data from previous systematic reviews with meta-analysis that show that women in general rate similar pain stimuli as more painful (47) and are more sensitive to pain than men (48).

In accordance with previous studies, there was no adverse effects by the injected solutions. Hypertonic saline and isotonic saline have previously been used in other studies without any side effects (31). Since sterile water often is used to dilute hypertonic saline, no side effects were anticipated. The pain induced by the injected solutions had vanished after a few minutes and had no long-lasting effects. In animal studies on rabbits and rodents, sterile water showed the less tissue damage than isotonic saline (26)

Some strengths and limitations of this study need to be addressed. One strength was that the experiment used a randomized, double blind and placebo controlled design which is considered to be the "gold standard" of human experimental studies (49). Also, using both a passive and an active control substance verifies the outcome of sterile water. Furthermore, the one-week wash out in-between sessions allowed the muscle tissue to restore in order not to cause any false pain experiences by remaining tissue damage. Another strength is that the injection depth in the masseter muscle was controlled so placement and depth of the needle could not affect the results. A limitation of this study was that the menstrual cycle for women was not considered. However, all women participating in the experiment were in different phases of the menstrual cycle and thus not likely to affect the results.

\section{Conclusions}

The study has shown that intramuscular injection of sterile water into the masseter muscle of young healthy men and women evokes pain with similar characteristic as hypertonic saline. Yet, there were no changes in PPT. Hence, in some respects the model mimics chronic myalgia and may offer a novel and simpler alternative to hypertonic saline injections. However, more studies are needed to further validate the results. It would also give a more in-depth knowledge of the pain mechanism of sterile water.

\section{Abbrevations}

$\mathrm{TMD}=$ temporomandibular disorders

$\mathrm{SD}=$ Standard deviation

VAS = Visual analogue scale

PPT = pressure pain threshold 
DC/TMD = Diagnostic Criteria for temporomandibular disorders

PSS-10 = Perceived Stress Scale

PHQ-9 = Patient Health Questionnaire 9

PCS = Pain Catastrophizing Scale

GAD-7 = Generalized Anxiety Disorder

PHQ-15 = Patient Health Questionnaire 15

\section{Declarations}

\section{Ethics approval and consent to participate}

The study followed the present guidelines according to the Declaration of Helsinki and was approved by the Regional Ethical Review Board in Stockholm, Sweden (2019/3:1). Verbal and written information of the study was provided to all participants and their written consent was obtained before the start of study.

\section{Consent for publication}

Not applicable.

\section{Availability of data and materials}

The datasets used and/or analysed during the current study are available from the corresponding author on reasonable request.

\section{Competing interests}

The authors declare that they have no competing interests. The authors alone are responsible for the content and writing of the paper. All authors have read and approved the final version of the manuscript.

\section{Funding}

Not applicable.

\section{Authors' contributions}

\section{Sofia Louca Jounger}

Responsible for data collection, contributed in the statistical analysis, and is the main author of the manuscript.

Johanna Svedenlöf

Contributed in the data collection, contributed in the statistical analysis, and in writing of the manuscript in general. 
Reija Elenius

Contributed in the data collection, contributed in the statistical analysis, and in writing of the manuscript in general.

\section{Christoffer Källkrans}

Contributed in the data collection, contributed in the statistical analysis, and in writing of the manuscript in general.

\section{Emil Scheid}

Contributed in the data collection, contributed in the statistical analysis, and in writing of the manuscript in general.

\section{Malin Ernberg}

Responsible to the design of the study, contributed to the statistical analysis, and in writing and revising the manuscript in general.

\section{Nikolaos Christidis}

Responsible to the design of the study, Responsible for data collection, the statistical analysis and in writing and revising the manuscript in general.

\section{Acknowledgements}

Not applicable.

\section{References}

1. Treede RD, Rief W, Barke A, Aziz Q, Bennett MI, Benoliel R et al (2015) A classification of chronic pain for ICD-11. Pain 156(6):1003-1007

2. Chisnoiu AM, Picos AM, Popa S, Chisnoiu PD, Lascu L, Picos A et al (2015) Factors involved in the etiology of temporomandibular disorders - a literature review. Clujul Med 88(4):473-478

3. Schiffman E, Ohrbach R, Truelove E, Look J, Anderson G, Goulet JP et al (2014) Diagnostic Criteria for Temporomandibular Disorders (DC/TMD) for Clinical and Research Applications: recommendations of the International RDC/TMD Consortium Network* and Orofacial Pain Special Interest Groupdagger. J Oral Facial Pain Headache 28(1):6-27

4. Lovgren A, Haggman-Henrikson B, Visscher CM, Lobbezoo F, Marklund S, Wanman A (2016) Temporomandibular pain and jaw dysfunction at different ages covering the lifespan-A population based study. Eur J Pain 20(4):532-540

5. Wright EF, North SL (2009) Management and treatment of temporomandibular disorders: a clinical perspective. J Man Manip Ther 17(4):247-254 
6. Breivik H, Collett B, Ventafridda V, Cohen R, Gallacher D (2006) Survey of chronic pain in Europe: prevalence, impact on daily life, and treatment. Eur J Pain 10(4):287-333

7. Dao TT, LeResche L (2000) Gender differences in pain. J Orofac Pain 14(3):169-184; discussion 84-95

8. Unell L, Johansson A, Carlsson GE, Halling A, Soderfeldt B (2006) Changes in reported orofacial symptoms over a ten-year period as reflected in two cohorts of fifty-year-old subjects. Acta Odontol Scand 64(4):202-208

9. Wadhwa S, Kapila S (2008) TMJ disorders: future innovations in diagnostics and therapeutics. J Dent Educ 72(8):930-947

10. Graven-Nielsen T (2006) Fundamentals of muscle pain, referred pain, and deep tissue hyperalgesia. Scand J Rheumatol Suppl 122:1-43

11. Louca S, Christidis N, Ghafouri B, Gerdle B, Svensson P, List T et al (2014) Serotonin, glutamate and glycerol are released after the injection of hypertonic saline into human masseter muscles - $\mathrm{a}$ microdialysis study. J Headache Pain 15:89

12. Le Bars D, Gozariu M, Cadden SW (2001) Animal models of nociception. Pharmacol Rev 53(4):597652

13. Graven-Nielsen T, Mense S (2001) The peripheral apparatus of muscle pain: evidence from animal and human studies. Clin J Pain 17(1):2-10

14. Reddy KS, Naidu MU, Rani PU, Rao TR (2012) Human experimental pain models: A review of standardized methods in drug development. J Res Med Sci 17(6):587-595

15. Graven-Nielsen T, Arendt-Nielsen L (2003) Induction and assessment of muscle pain, referred pain, and muscular hyperalgesia. Curr Pain Headache Rep 7(6):443-451

16. Svensson P, Cairns BE, Wang K, Hu JW, Graven-Nielsen T, Arendt-Nielsen L et al (2003) Glutamateevoked pain and mechanical allodynia in the human masseter muscle. Pain 101(3):221-227

17. Louca S, Ernberg M, Christidis N (2013) Influence of intramuscular granisetron on experimentally induced muscle pain by acidic saline. J Oral Rehabil 40(6):403-412

18. Babenko VV, Graven-Nielsen T, Svensson P, Drewes AM, Jensen TS, Arendt-Nielsen L (1999) Experimental human muscle pain induced by intramuscular injections of bradykinin, serotonin, and substance P. Eur J Pain 3(2):93-102

19. Christidis N, loannidou K, Milosevic M, Segerdahl M, Ernberg M (2008) Changes of hypertonic salineinduced masseter muscle pain characteristics, by an infusion of the serotonin receptor type 3 antagonist granisetron. J Pain 9(10):892-901

20. Martensson L, Stener-Victorin E, Wallin G (2008) Acupuncture versus subcutaneous injections of sterile water as treatment for labour pain. Acta Obstet Gynecol Scand 87(2):171-177

21. Martensson L, Nyberg K, Wallin G (2000) Subcutaneous versus intracutaneous injections of sterile water for labour analgesia: a comparison of perceived pain during administration. BJOG 107(10):1248-1251 
22. Cui JZ, Geng ZS, Zhang YH, Feng JY, Zhu P, Zhang XB. Effects of intracutaneous injections of sterile water in patients with acute low back pain: a randomized, controlled, clinical trial. Braz J Med Biol Res. 2016;49(3)

23. Le Bars D, Dickenson AH, Besson JM (1979) Diffuse noxious inhibitory controls (DNIC). I. Effects on dorsal horn convergent neurones in the rat. Pain 6(3):283-304

24. Wreje U, Brorsson B (1995) A Multicenter Randomized Controlled Trial of Injections of Sterile Water and Saline for Chronic Myofascial Pain Syndromes. Pain 61(3):441-444

25. Saxena KN, Nischal H, Batra S (2009) Intracutaneous Injections of Sterile Water over the Secrum for Labour Analgesia. Indian J Anaesth 53(2):169-173

26. Comereski CR, Williams PD, Bregman CL, Hottendorf GH (1986) Pain on injection and muscle irritation: a comparison of animal models for assessing parenteral antibiotics. Fundam Appl Toxicol 6(2):335-338

27. Riley JL 3rd, Robinson ME, Wise EA, Myers CD, Fillingim RB (1998) Sex differences in the perception of noxious experimental stimuli: a meta-analysis. Pain 74(2-3):181-187

28. DiBartola SP (2012) Chapter 3 - Disorders of Sodium and Water: Hypernatremia and Hyponatremia. In: Fluid, Electrolyte, and Acid-Base Disorders in Small Animal Practice (Fourth Edition). W.B. Saunders, Saint Louis, pp 45-79

29. Hawker GA, Mian S, Kendzerska T, French M (2011) Measures of adult pain: Visual Analog Scale for Pain (VAS Pain), Numeric Rating Scale for Pain (NRS Pain), McGill Pain Questionnaire (MPQ), ShortForm McGill Pain Questionnaire (SF-MPQ), Chronic Pain Grade Scale (CPGS), Short Form-36 Bodily Pain Scale (SF-36 BPS), and Measure of Intermittent and Constant Osteoarthritis Pain (ICOAP). Arthritis Care Res (Hoboken) 63(Suppl 11):S240-S252

30. Veerasarn P, Stohler CS (1992) The effect of experimental muscle pain on the background electrical brain activity. Pain 49(3):349-360

31. Christidis N, Loannidou K, Mllosevic M, Segerdahl M, Ernberg M (2008) Changes of Hypertonic Saline-Induced Masseter Muscle Pain Characteristics, by an Infusion of the Serotonin Receptor Type 3 Antagonist Granisetron. J Pain 9(10):892-901

32. Svensson P, List T, Hector G (2001) Analysis of stimulus-evoked pain in patients with myofascial temporomandibular pain disorders. Pain 92(3):399-409

33. Graven-Nielsen T, Arendt-Nielsen $L$ (2003) Induction and assessment of muscle pain, referred pain, and muscular hyperalgesia. Curr Pain Headache Rep 7(6):443-451

34. Louca Jounger S, Christidis N, Hedenberg-Magnusson B, List T, Svensson P, Schalling M et al (2016) Influence of Polymorphisms in the HTR3A and HTR3B Genes on Experimental Pain and the Effect of the 5-HT3 Antagonist Granisetron. PLoS One 11(12):e0168703

35. Graven-Nielsen T, Arendt-Nielsen L, Svensson P, Jensen TS (1996) Experimental muscle pain: a quantitative study of local and referred pain in humans following injection of hypertonic saline. $J$ Musculoskel Pain 5(1):49-69 
36. Graven-Nielsen T, Babenko V, Svensson P, Arendt-Nielsen L (1998) Experimentally induced muscle pain induces hypoalgesia in heterotopic deep tissues, but not in homotopic deep tissues. Brain research 787:203-210

37. Svensson P, Wang K, Arendt-Nielsen L (2003) Effect of muscle relaxants on experimental jaw-muscle pain and jaw-stretch reflexes: a double-blind and placebo-controlled trial. Eur J Pain (London England) 7(5):449-456

38. Tegeder L, Zimmermann J, Meller ST, Geisslinger G (2002) Release of algesic substances in human experimental muscle pain. Inflamm Res 51(8):393-402

39. Graven-Nielsen T, McArdle A, Phoenix J, Arendt-Nielsen L, Jensen TS, Jackson MJ et al (1997) In vivo model of muscle pain: quantification of intramuscular chemical, electrical, and pressure changes associated with saline-induced muscle pain in humans. Pain 69(1-2):137-143

40. Lindahl O (1969) Experimental muscle pain produced by chemical stimulus. Acta Orthop Scand 40(6):741-750

41. Kiil F (1982) Mechanism of osmosis. Kidney Int 21(2):303-308

42. Hoffmann EK, Lambert IH, Simonsen LO (1986) Separate, Ca2+-activated K + and Cl- transport pathways in Ehrlich ascites tumor cells. J Membr Biol 91(3):227-244

43. Christidis N, loannidou K, Milosevic M, Segerdahl M, Ernberg M (2008) Changes of hypertonic salineinduced masseter muscle pain characteristics, by an infusion of the serotonin receptor type 3 antagonist granisetron. J Pain: official journal of the American Pain Society 9(10):892-901

44. Cairns BE, Hu JW, Arendt-Nielsen L, Sessle BJ, Svensson P (2001) Sex-related differences in human pain and rat afferent discharge evoked by injection of glutamate into the masseter muscle. J Neurophysiol 86(2):782-791

45. Komiyama O, Gracely RH, Kawara M, Laat AD (2008) Intraoral measurement of tactile and filamentprick pain threshold using shortened Semmes-Weinstein monofilaments. Clin J Pain 24(1):16-21

46. Christidis N, Kopp S, Ernberg M (2005) The effect on mechanical pain threshold over human muscles by oral administration of granisetron and diclofenac-sodium. Pain 113(3):265-270

47. Berkley KJ. Sex differences in pain. Behav. Brain Sci. 1997;20:371 - 80 discussion 435-513.

48. Fillingim RB, King CD, Ribeiro-Dasilva MC, Rahim-Williams B, Riley JL 3 (2009) Sex, gender, and pain: a review of recent clinical and experimental findings. J Pain: official journal of the American Pain Society 10(5):447-485 rd. .

49. Misra S (2012) Randomized double blind placebo control studies, the "Gold Standard" in intervention based studies. Indian J Sex Transm Dis AIDS 33(2):131-134

\section{Tables}

Table 1. Baseline demographic status of 15 healthy, pain-free women and 15 healthy and pain-free, age-matched men, i.e. before injection of any of the substances. 


\begin{tabular}{|c|c|c|}
\hline & Women $(n=15)$ & $\begin{array}{c}\text { Men } \\
(n=15)\end{array}$ \\
\hline \multicolumn{3}{|l|}{ Age } \\
\hline Mean (SD) & $23.5(1.9)$ & $23.7(2.9)$ \\
\hline Min-max & $20-28$ & $21-32$ \\
\hline \multicolumn{3}{|l|}{ Stress (PSS-10) } \\
\hline Median (IQR) & $15(7.5)$ & $15(4.2)$ \\
\hline No stress (0-12 points) & $\mathrm{n}=6$ & $\mathrm{n}=6$ \\
\hline Moderate degree of stress (13-20 points) & $\mathrm{n}=7$ & $\mathrm{n}=9$ \\
\hline Severe degree of stress (21-40 pints) & $\mathrm{n}=2$ & $\mathrm{n}=0$ \\
\hline \multicolumn{3}{|l|}{ Depression (PHQ-9) } \\
\hline Median (IQR) & $3(5.9)$ & $4(4.2)$ \\
\hline Normal (0-4 points) & $\mathrm{n}=8$ & $\mathrm{n}=9$ \\
\hline Mild (5-9 points) & $\mathrm{n}=6$ & $\mathrm{n}=4$ \\
\hline Moderate (10-14 points) & $\mathrm{n}=0$ & $\mathrm{n}=1$ \\
\hline Moderately severe (15-19 points) & $\mathrm{n}=0$ & $\mathrm{n}=1$ \\
\hline Severe (20-24 points) & $\mathrm{n}=1$ & $\mathrm{n}=0$ \\
\hline \multicolumn{3}{|l|}{ Pain catastrophizing (PCS) } \\
\hline Median (IQR) & $5(6.2)$ & $6(5.7)$ \\
\hline None (0-19 pints) & $\mathrm{n}=15$ & $\mathrm{n}=15$ \\
\hline Risk of clinical pain catastrophizing ( $20-29$ points) & $\mathrm{n}=0$ & $\mathrm{n}=0$ \\
\hline High risk of clinical pain catastrophizing ( $\geq 30$ points) & $\mathrm{n}=0$ & $\mathrm{n}=0$ \\
\hline \multicolumn{3}{|l|}{ Anxiety (GAD-7) } \\
\hline Median (IQR) & $2(4.6)$ & $3(2.9)$ \\
\hline Normal (0-4 points) & $\mathrm{n}=11$ & $\mathrm{n}=8$ \\
\hline Mild (5-9 points) & $\mathrm{n}=3$ & $\mathrm{n}=7$ \\
\hline Moderate (10-14 points) & $\mathrm{n}=0$ & $\mathrm{n}=0$ \\
\hline Severe (15-27 points) & $\mathrm{n}=1$ & $\mathrm{n}=0$ \\
\hline \multicolumn{3}{|l|}{ Somatization (PHQ-15) } \\
\hline Median (IQR) & $6(4.7)$ & $5(2.1)$ \\
\hline Normal (0-4 points) & $\mathrm{n}=6$ & $\mathrm{n}=5$ \\
\hline Mild (5-9 points) & $\mathrm{n}=5$ & $\mathrm{n}=10$ \\
\hline Moderate (10-14 points) & $\mathrm{n}=3$ & $\mathrm{n}=0$ \\
\hline Severe (15-30 points) & $\mathrm{n}=1$ & $\mathrm{n}=0$ \\
\hline
\end{tabular}

Data in the table are expressed as median (interquartile range; IQR: 75th percentile minus 25th percentile).

PSS-10 $=$ Perceived Stress Scale 
PHQ-9 = Patient Health Questionnaire 9

PCS = Pain Catastrophizing Scale

GAD-7 = Generalized Anxiety Disorder

PHQ-15 = Patient Health Questionnaire 15

. Table displaying median (IQR) peak pain intensity (VAS 0-100), pain duration (s), the area under the curve (VAS auc) and pain area (au) after ns of hypertonic saline, sterile water and isotonic saline (placebo) into the masseter muscle of 15 healthy women and 15 healthy and age-matched

\begin{tabular}{|c|c|c|c|}
\hline & Sterile water & Hypertonic saline & Isotonic saline \\
\hline \multicolumn{4}{|l|}{ Jain } \\
\hline & $74.5(49.9)^{\$}$ & $74.0(50.5)$ & $11.4(23.4)^{\#}$ \\
\hline Women & $74.5(46.9)$ & $74.0(50.5)$ & $11.5(23.4)$ \\
\hline Men & $65.0(39.0)$ & $66.5(19.0)$ & $6.0(33.0)$ \\
\hline$p$-value (men vs women) & 0.798 & 0.721 & 0.901 \\
\hline \multicolumn{4}{|l|}{ luration } \\
\hline & $660(889)^{\$}$ & $285(361)^{f}$ & $98(128){ }^{\#}$ \\
\hline Women & 490 (375) & $285(95)$ & $60(75)$ \\
\hline Men & $1260(1080)$ & $660(1110)$ & $120(310)$ \\
\hline$p$-value (men vs women) & 0.002 & 0.002 & 0.006 \\
\hline \multicolumn{4}{|l|}{ uc } \\
\hline & $1230.7(1815.0)$ & $814.5(1144.1)$ & $4.6(45.2)$ \\
\hline Women & $1213.6(1407.9)$ & $847.3(973.1)$ & $7.2(44.5)$ \\
\hline Men & $1790.7(2542.4)$ & $581.3(1227.1)$ & $0(83.24)$ \\
\hline$p$-value (men vs women) & 0.300 & 0.269 & 0.200 \\
\hline \multicolumn{4}{|l|}{ rea } \\
\hline & $194.6(177.4)^{\$}$ & $175.9(253.0)^{£}$ & $12.6(70.9)$ \# \\
\hline Women & $200.8(171.3)$ & 324.5 (239.4) & $37.2(61.6)$ \\
\hline Men & $173.3(204.5)$ & $99.4(110,1)$ & $5.7(88.3)$ \\
\hline$p$-value (men vs women) & 0.281 & 0.003 & 0.368 \\
\hline
\end{tabular}

nterquartile range (IQR: 75th percentile minus 25th percentile).

ïcant difference between hypertonic saline and isotonic saline, ${ }^{£}$ between hypertonic saline and sterile water, and ${ }^{\$}$ between sterile water and saline (Friedman ANOVA with Tukey post hoc test, $P<0.05$ ).

ures denote significant difference between sexes $(P<0.05)$ 


\section{Figures}

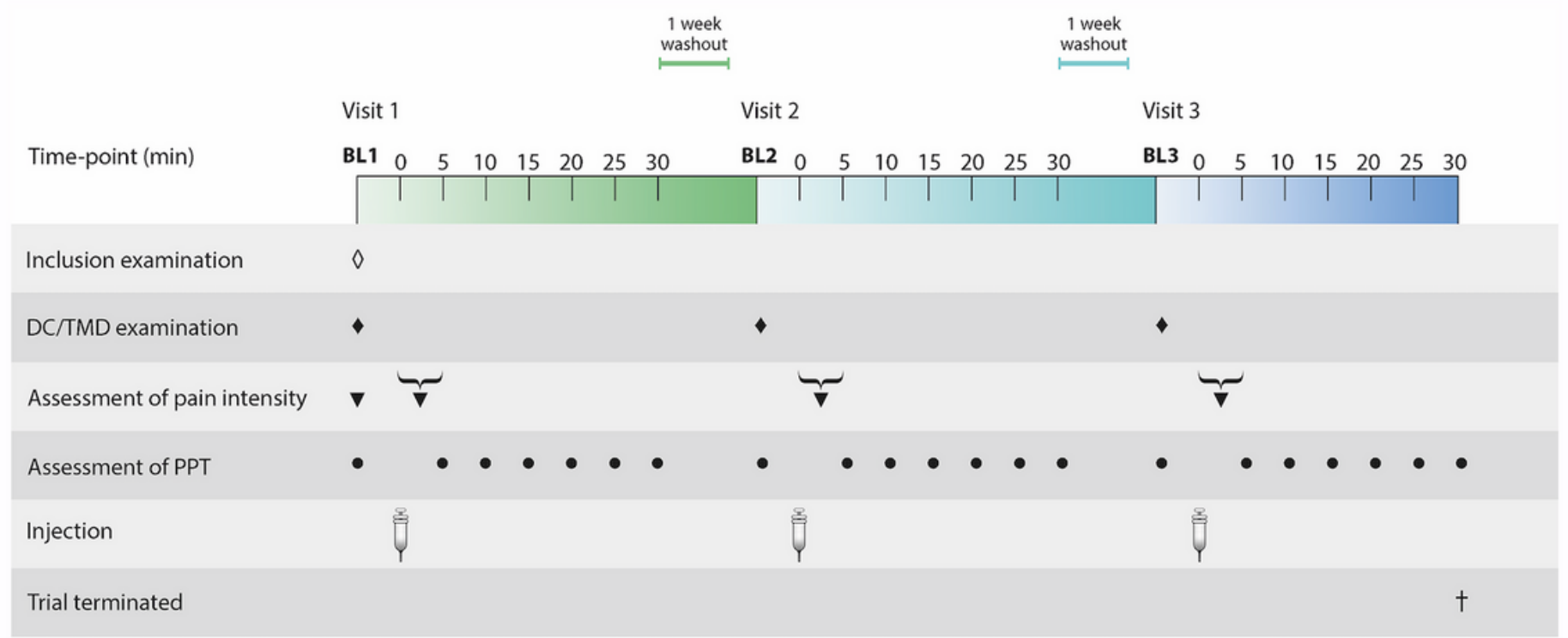

\section{Figure 1}

Flowchart displaying the study protocol. The flowchart shows the time-points in minutes for every registration: inclusion examination, baseline (BL1-3) for each session, and the experimental assessments of pain intensity (VAS; visual analogue scale), pressure pain threshold (PPT), pain area as well as the time-points for injections of test substances, in 15 healthy, pain-free women and in 15 healthy, pain-free and age-matched men. 


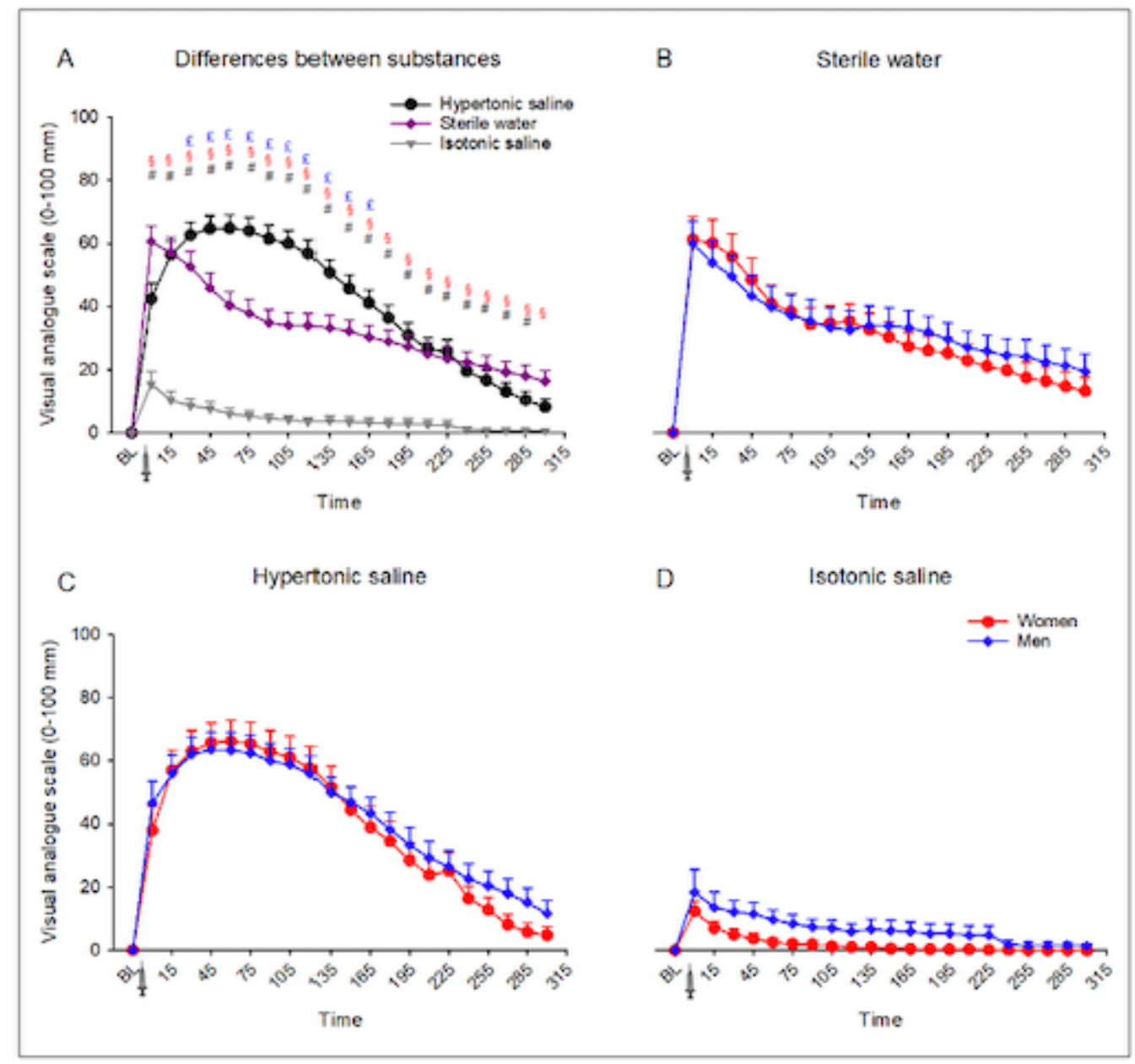

Figure 2

The mean (SEM) changes in pain intensity (VAS; visual analogue scale) compared with baseline (BL) after injections with the test substances hypertonic saline, isotonic saline and sterile water in 30 healthy, pain-free participants (A) and divided by sex into 15 women and 15 age-matched men (B-D).

Assessments displayed were made every 15 th $\mathrm{s}$ beginning immediately after injection up to $300 \mathrm{~s}$ after injection. The changes in pain intensity are presented both for the entire group and for the sexes separately. \# Significant difference between hypertonic and isotonic saline § Significant difference between sterile water and isotonic saline $£$ Significant difference between hypertonic saline and sterile water The level of significance is set to $P<0.05$. 


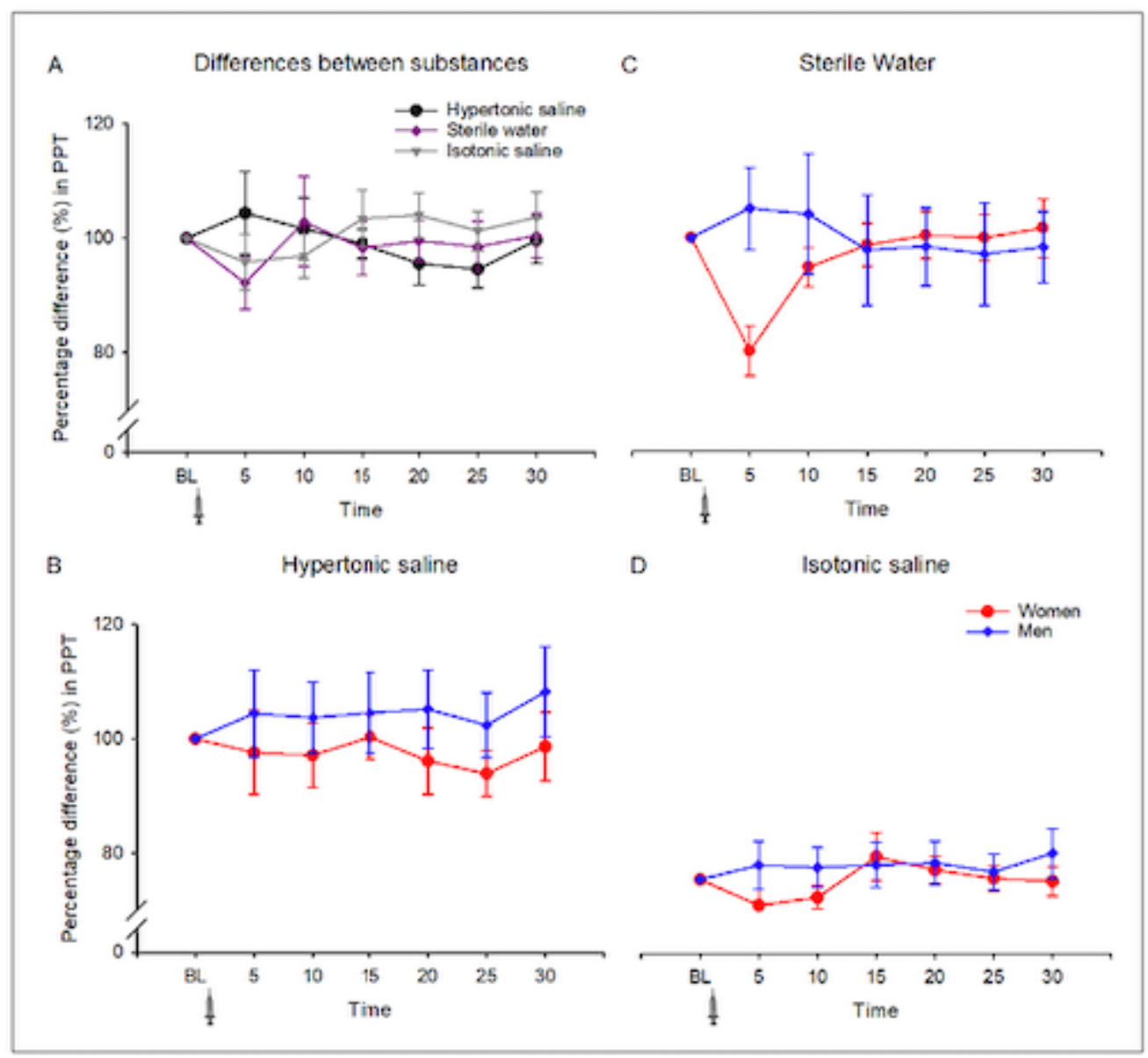

\section{Figure 3}

The mean (SEM) percentage changes in pressure pain threshold (PPT; $\mathrm{kPa}$ ) to baseline (BL) after intramuscular injections of hypertonic saline, isotonic saline and sterile water in 30 healthy, pain-free participants (A) and divided by sex into 15 women and 15 age-matched men (B-D). Assessments displayed were made every 5th min beginning $5 \mathrm{~min}$ after injection up to $30 \mathrm{~min}$ after injection. The changes in PPT are presented both for the entire group and for the sexes separately. Part A) display the percentage change in PPT over the masseter muscle. There were no significant differences in PPT over time or between substances. When sexes were analyzed separately, PPT was significantly lower in the women compared to baseline 5 min after injection with hypertonic saline (Tukey test). Part B) display the percentage change in PPT over the reference point (the right index fingertip). There were no significant differences in PPT over time nor any between substances for the entire group, or when the sexes were analyzed separately. The level of significance is set to $P<0.05$. 


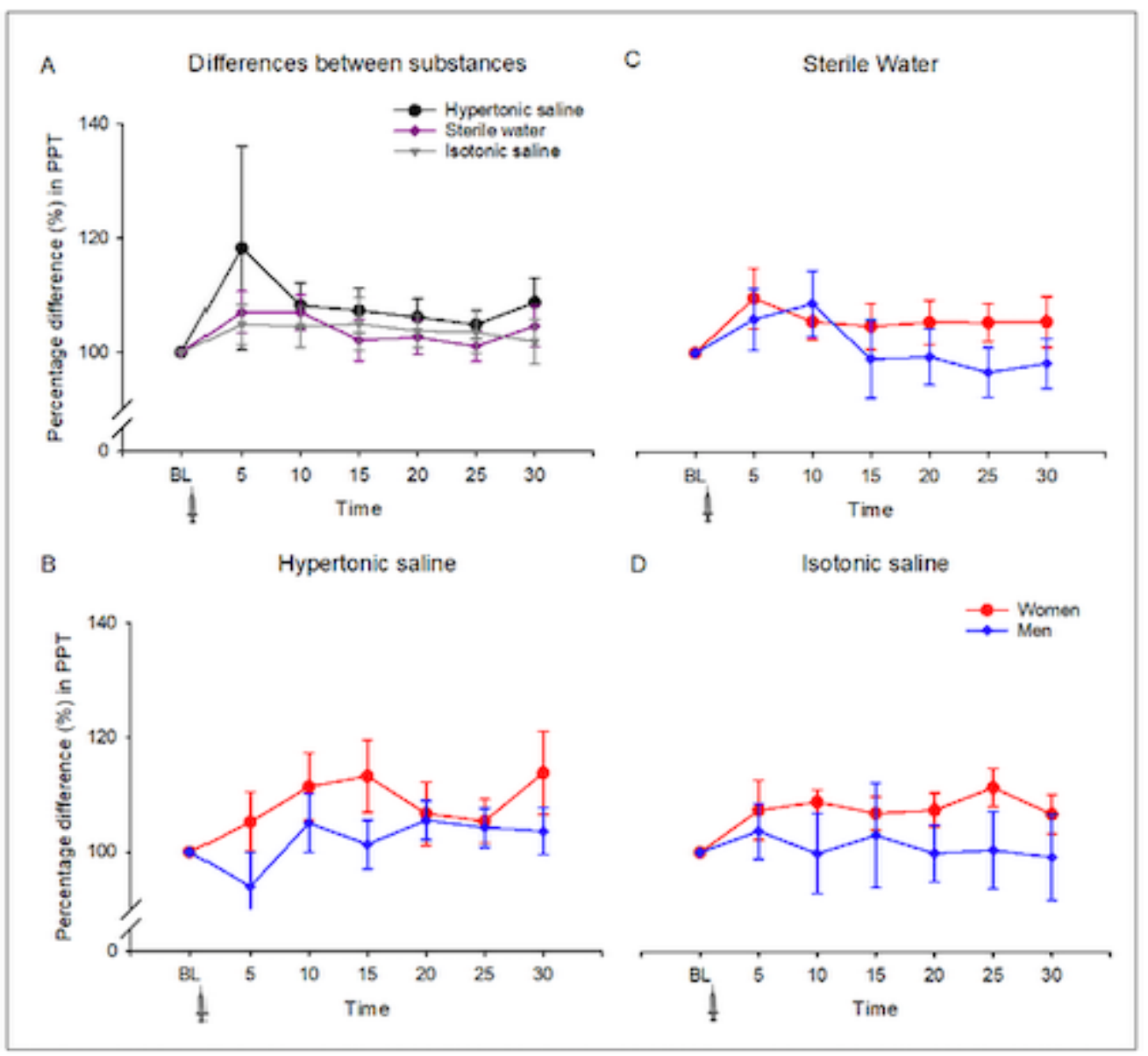

Figure 4

A legend was omitted by the authors in this version of the paper. 